\title{
A MODEL STUDY OF SMALL-SCALE WORLD MAP GENERALIZATION
}

\author{
Y. Cheng ${ }^{1}$, Y. Yin ${ }^{1}$, C.M.L ${ }^{1}$, W. Wu ${ }^{1}$, P.P. Guo ${ }^{1}$, X. L. Ma ${ }^{1}$, F.M.Hu \\ ${ }^{1}$ Institute of Cartography and Geographic Information System, Chinese Academy of Surveying and Mapping, Beijing, China. \\ 1435569554@qq.com,yinyong@casm.ac.cn,cmli@casm.ac.cn, zzww1016@126.com, guopeipei925@163.com, \\ 1510912@tongji.edu.cn \\ ${ }^{2}$ School of Remote Sensing and Information Engineering, Wuhan University. - hfm2014@163.com
}

\section{Commission III, WG III/4}

\begin{abstract}
KEY WORDS: Global map, Map generalization, Improved model, Cross-platform symbol, Automatic map-making knowledge
\end{abstract} engine

\begin{abstract}
:
With the globalization and rapid development every filed is taking an increasing interest in physical geography and human economics. There is a surging demand for small scale world map in large formats all over the world. Further study of automated mapping technology, especially the realization of small scale production on a large scale global map, is the key of the cartographic field need to solve.In light of this, this paper adopts the improved model (with the map and data separated) in the field of the mapmaking generalization, which can separate geographic data from mapping data from maps, mainly including cross-platform symbols and automatic map-making knowledge engine. With respect to the cross-platform symbol library, the symbol and the physical symbol in the geographic information are configured at all scale levels. With respect to automatic map-making knowledge engine consists 97 types, 1086 subtypes, 21845 basic algorithm and over 2500 relevant functional modules.In order to evaluate the accuracy and visual effect of our model towards topographic maps and thematic maps, we take the world map generalization in small scale as an example. After mapping generalization process, combining and simplifying the scattered islands make the map more explicit at 1:2.1 billion scale, and the map features more complete and accurate. Not only it enhance the map generalization of various scales significantly, but achieve the integration among map-makings of various scales, suggesting that this model provide a reference in cartographic generalization for various scales.
\end{abstract}

\section{INTRODUCTION}

In the recent times, surveying and mapping technology has gained increasingly wide application owing to the rapid development of science and technology. Map Generalization is an important means for obtaining basic geographic information on different measurement scales, laying the foundation for rational applications of survey results and providing more convenient services for surveying, mapping, and production. With the current technology, most existing results in the field of map generalization have required manual or semi-automatic intervention. Processing speeds and accuracy cannot meet the current demand, and they lack unified specifications for map integration. There is an urgent need to resolve problems, such as improving the efficiency of map generalization, effectively shorten the time required for cross-scale charting, and improve the automation of map integration, which has been world problems for more than half century.

Currently, various kinds of charting software and GIS software exist. Most contain functional modules for the comprehensive integration of maps. However, these modules are scattered and lack ideal solution strategies, and the theoretical systems, methods, and procedures, and algorithm interfaces are not consistent. Because of the complexity of data on different scales, the workload in the later manual-processing stage is greatly increased. In foreign markets, GIS software such as ARC/INFO produced by ESRI employs map making just as a software output function, while the analysis of spatial data is a core function. This weakened map-making function fails to meet the needs of more formal map publication. In the domestic market, both GIS mapping software and the SHANHAIYIHUI software employ a relatively low degree of automation. They are basically human-computer interaction methods, which can only process a single map containing a small amount of data. If they are widely applied to the large amounts of map data, the process of map generation and type setting will both be delayed.

To overcome such problems, the WJ-III team at the Chinese Academy of Surveying and Mapping has proposed a new concept of information-based infinite focus-change mapping, with a goal of organically integrating cartographic software having proprietary intellectual property rights with computer hardware to strengthen cartographic visualization and efficiency. This approach not only greatly improves the traditional map data productivity at a single scale and with fixed focus but also is highly efficient in producing map data on arbitrary scales and with variable focal lengths. Relying on the automated mapping process in the work station, it can make the entire process of map making $100 \%$ digitized and more than $90 \%$ automated, improving map-making efficiency by more than a factor of five. Using the software in the work station and by studying related materials of the world map at a relatively big scale span, this article uses the example of generalizing the scale of measurement from 1:5 million to 1:200 million to introduce in detail the figure and number separation model and related algorithms in the work station and to provide a specific example of cross-scale integration and automated map making.

\section{DATA INTRODUCTION}

\subsection{Data Source}

Surface data include data from national administrative regions worldwide, as well as for oceans and rivers that cover relatively large areas. The basic data is in GDB database format. 
Line data include the international date line annotations along the international date line, line data for rivers, land-boundary data for each state, national (or regional) boundaries (including both determined and undetermined boundaries, military demarcation lines, cease-fire lines, territorial boundaries claimed by the islands, and maritime boundaries, among others) The data also include the main rivers and lakes in the world (class I rivers in China and class I and II rivers and lakes abroad are shown in the minimum scale). Some rivers are represented by a single line, some by center line, and some by riverstructure lines; all are in vector shp format.

Line data also include island data, including data for the South China Sea Islands Diaoyu Dao and Chiwei Yu, among others, and all data are in vector shp format.

\subsection{Study Scope}

This article adopts the internationally accepted world map of the Pacific Ocean using the $30^{\circ} \mathrm{W}$ meridian in the Atlantic Ocean as the split line. This is also known as "Pacific Ocean Version World Map." It retains the world time zones, guarantees minimum deformations in the drawings, and adheres to the principle of dividing the land as little as possible. This model also shows the overall geographical relations among different plates in the world. In addition to retaining the integrity of all the continents in the world, it centralizes the position of China To focus on the characteristics of map integration, this study does not focus on the administrative regions such as national boundary lines, especially for politically sensitive regions.

\section{APPROACHES}

\subsection{Globally Oriented, Small-Scale Figure-Number Separation Model}

A figure-number separation model can be used to integrate geographical data and graphic data separately in a map. The model we use includes a cross-platform symbol library and an auto-drafting knowledge engine. The cross-platform symbol library is equipped with graphic symbols for various levels of measuring scales as well as entity symbols for geographic information. Currently, the symbol library is equipped with graphic symbols for engineering applications in 10 areas, including Inner Mongolia, Jilin, Liaoning, Hebei, Sichuan, and Guizhou, among others. The auto-drafting knowledge engine includes 97 classes, 1,086 subclasses, and 21,845 basic algorithms, and its related functional module can reach more than 2,500 types. In many provinces and cities, geographic map making is currently a problem for departments and organizations. The auto-drafting knowledge engine we used has previously been employed to help Chishui and Guizhou to integrate eight classes of geographical information and to solve the problems of combining, simplifying, splitting, and integrating 10 classes and more than 50 figure spots. The crossplatform symbol library and auto-drafting knowledge engine system are illustrated in Fig. 1.

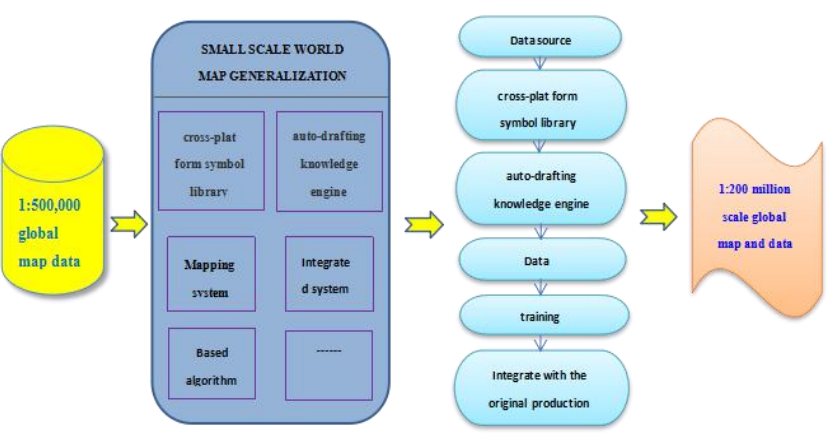

Figure 1. Architecture of the Figure-Number Separation Model

\subsection{Key Algorithms}

To take advantage of the features and architecture of the figurenumber separation model, this study primarily uses the basic algorithms in the WJ-III mapping work station, including those dealing with topology (such as topology generation and topology updating, among others), triangulation networks (also known as Delaunay triangulation networks), and geometrical computations (such as calculations of length and area, among others). The work station not only includes algorithms for integrating world-map plate areas across different measuring scales but also includes algorithms to determine water-system gradients from river lines and integrated water-system surfaces, thus enabling cartographic generalizations from measuring scales of 1:5 million to 1: 200 million.

\subsubsection{Geometrical Simplifications to Retain Topological Relations}

3.2.1.1 Construction of Topological Relations: Topological relations are qualitative types of spatial information that do not change with spatial rotation, translation, and zoom and scaling, among others. To maintain continuity in a map, the contiguity, inclusion, intersections, and geometric types of spatial targets i.e., the feature type of a spot, line, or surface, among others, must remain unchanged. We need to construct the topological relations of the original data to maintain the same topological relations when simplifying figure-spots.

3.2.1.2 Geometric Simplification of a World Map that Maintain Topological Relations: In process of integrating ordinary topographic maps, feature classes of various polygonal shapes are usually scattered and independent, and a polygon is generally constructed of line segments that are saved in a continuous coordinate sequence. Generally, special simplifications are not needed for figure spots. Instead, we use a general curve-simplification algorithm. But in the integration process of a small-scale world map, the spatial distribution of the land cover is characterized by seamlessness with no overlap, and each plate carries its own semantic information. Simplifying each plate boundary one by one may generate gaps or overlapping fragments between adjacent figure spots, making the shared boundary inconsistent and failing to maintain the topological relations between figure spots. Therefore, we cannot use ordinary simplification methods for normal curves directly to treat plate boundaries. Instead, we need to take the approach of geometrical simplification, which maintains the topological relationships. In geometrical simplification, to maintain topological relations, we perform topology construction using the Li-Openshaw technique(Feng,et al.,2017) to simplify each shared arc. In the world-map integration process, the boundary of China, for example, is defined to be a restricted arc, which is 
not subjected to simplification. The detailed process is as follows:

1. Pretreat the original world-map plate data, checking and rectifying topological relations among the data on each plate to guarantee that there is no overlap and that the plate data is seamless.

2. Conduct topological construction as described above and check the construction results. Divide arcs according to the attribute information of the plate data Divide topological segments from non-topological segments.

3. Determine whether or not a constructed topological segment is restricted. If the segment is restricted, allow no simplification. If the segment is nonrestricted, adopt the Li-openshaw method to simplify it.

Traverse all nonrestrictive arcs and conduct geometric simplifications of all plate-surface data to retain topological relations.

\subsubsection{Gradual Changes of Water Systems}

River systems are important parts of the world map. They are shown by tapered lines that gradually turn bold as one proceeds downriver. Since world-map river data include surface data, double-line data, and single-line data, the gradual changes along a river on the world map mainly involve the following three aspects: 1) extraction of the central axis of a river, 2) conversion of a double-line river to a single-line river, and 3) determining the hierarchical relations in a river system.

3.2.2.1 Extraction of the Central Axis of a River: The key to central-axis extraction for a river is to search for equidistant points between an interior polygon and boundary line. In essence, this requires an analysis of spatial adjacency. The Delaunay triangulation network is a useful tool for exploring adjacency relations in a space graph, and this article employs Delaunay triangulation to extract the central axis of a river.

Delaunay triangulation extracts the central axis for three kinds of triangles using the methods described below and connects the central-axis points to form a skeleton line. The common border between two adjacent triangles is called an "adjacent edge."

Class I triangle: Connect the midpoint of the only adjacent edge and its related apex, such as the segments AH, DJ, and FK in Fig. 2.

Class II triangle: Connect the midpoints of two adjacent edges such as segment IJ in Fig. 2.

Class III triangle: Connect the midpoints of the center of gravity and the three sides, such as segments LI, LK, and LH in Fig. 2.

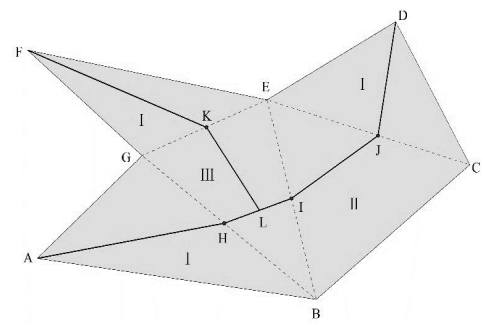

Figure 2. Triangle Classifications

This article adopts the algorithm of central-axis extraction based on the Delaunay triangle network, the detailed steps for which are listed in reference (Ai, et al.,2000). Line-boundary chattering generates redundant central-axis branches of a river. Ai Tinghua approached this problem by (1) abandoning triangles for which all three points are in the same polygonal boundary and discarding the corresponding parts of the central axis; and (2) setting a length threshold. It is reasonable to abandon a central-axis branch unless it exceeds a threshold value. However, for a river, all points share the same polygonal boundary. Consequently, we abandon method (1) and instead adopt method (2).

3.2.2.2 Converting a Double-Line River to a Single-Line River: Double-line rivers are divided into two types; one connects to a single-line river, while the other does not. For a double-line river that does not connect to single-line river, we adopt the method of central-line extraction described above. When a double-line river does connect to single-line river, we extract the central axis and connect it with the single-line river. In Fig. 3, A and B are two single-line rivers. Make triangle network to Double-line rivers, the central axis ACDB of the double-line river guarantees connectivity with the two singleline rivers.

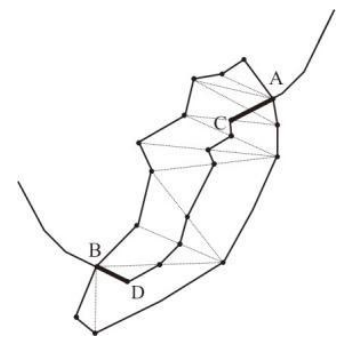

Figure 3. Central Axis Extraction for a Double-Line River

3.2.2.3 Study of the Hierarchical Relationships in a River System: River systems on a map are complex and have multiple forms. However, most river systems have a tree structure, i.e., there exists an apparent hierarchy of mainstream and tributaries with a "parent-child relationship" among them (see Fig. 4).

Stroke derives from the good continuation in Gestalt cognition principle. This concept stems from the thought of drawing a curve section at one stroke (Liu, et al., 2010; Yang,et al., 2013). In this article, we start from the mouth of the river and proceed upstream from the downstream river mouth, where it enters the sea. As discussed in more detail below, we consider river semantics, lengths of branches, and directional constraints iteratively to construct a tree-like river system of stroke connections to determine the hierarchical relations within a river system. The detailed process for constructing the hierarchical relations within a river system is discussed in (Wu, et al.,2016).

To determine the node that represents the mouth of the river the degree of correlation of the node must equal 1, where the degree of correlation refers to the number of segment arcs connected to a node. The node is at the end of a segment. The first node of node correlation section arc is the end node of other correlation segment arc of the first node. The node intersects with sea level. 


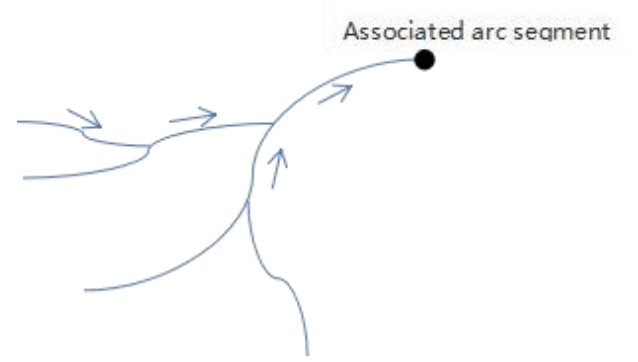

Figure 4.River mouth

Basic Principles of Stroke Connections: The following points are illustrated in Fig. 5:

(1) Semantic consistency: The feature ID and name ID of a segmental arc in the same river must be consistent. Therefore, the same strokes are to be connected to a segmental river arc having the same name. A perennial river, a dry riverbed, intermittent river, and other kinds of segmental river arcs are combined following the principles of length priority and directional consistency to build stroke connections.

(2) Length priority: The length of a river segment is the secondmost important factor to consider in constructing stroke connections after river-system semantics. The longest segment arc is to be connected first at a river fork.

(3) Directional consistency: The adjacent segment must conform to the principle of benign continuity, and the connection transition must be a natural one. The closer the connecting angle is to $180^{\circ}$, the more difficult it becomes to build a stroke connection. Rivers with opposing flow directions cannot be connected into a single stroke.

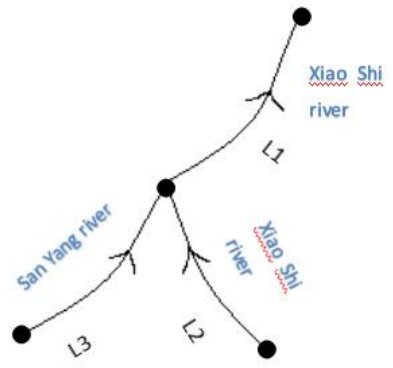

(a)

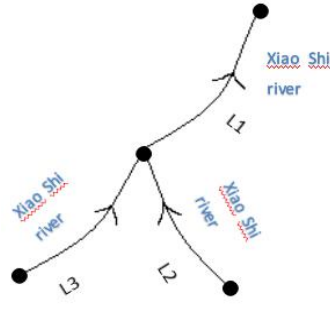

(b)

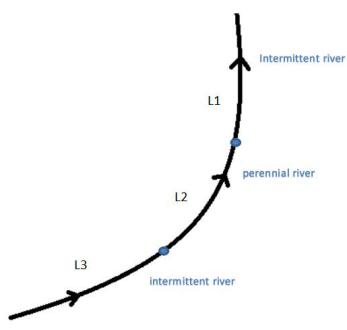

(c) (a) Semantic Consistency Principle (b) Length Priority Principle (c) Directional Consistency Principle Figure 5. Stroke Construction Method

\section{EXAMPLE OF THE ANALYSIS}

\subsection{Plate Integration}

To integrate separate plates into a single map, we adopt geometrical simplifications to maintain topological relations, keeping the original boundary shapes and geospatial relations of the plates unchanged and maintaining the basic semantic information for each plate. After simplifying the plate surface, it is compulsory to connect to the boundaries of the water system, to keep the plate and river lines seamlessly connected and maintain the original topological shapes. If the map is just to be used for display on an e-government platform, it is not necessary to retain national boundaries. To highlight China, however, we retain the borders of China and conduct no simplification. We also retain all ten section lines of the South China Sea, and we do not change the treatment of any island neighboring China.

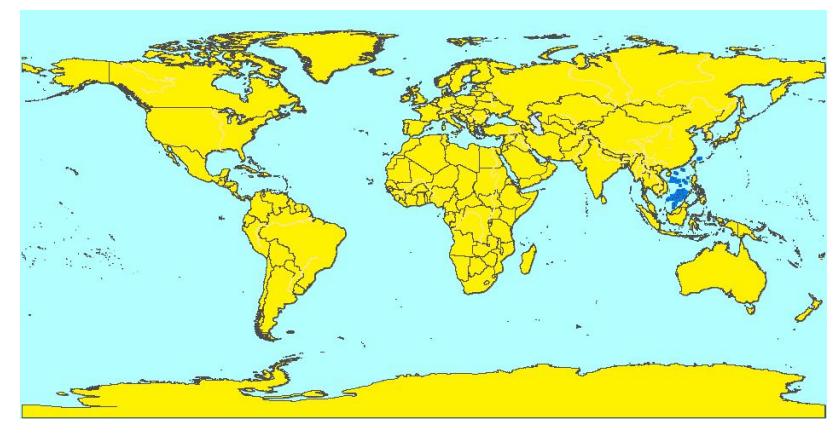

Figure 6. Before Plate Generalization

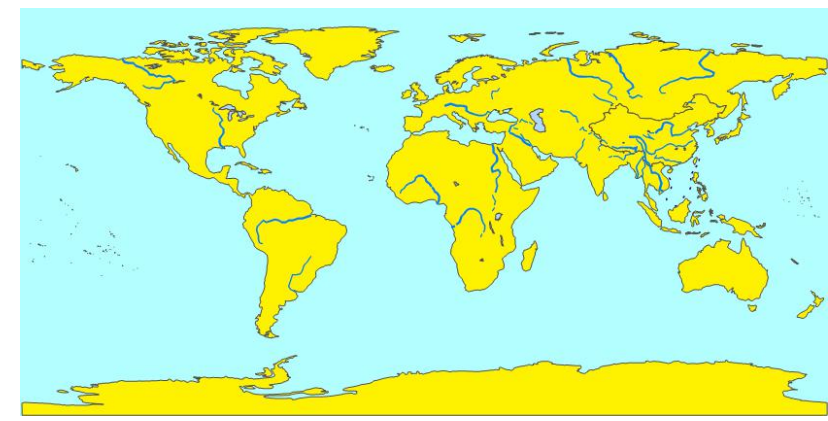

Figure 7. After Plate Generalization
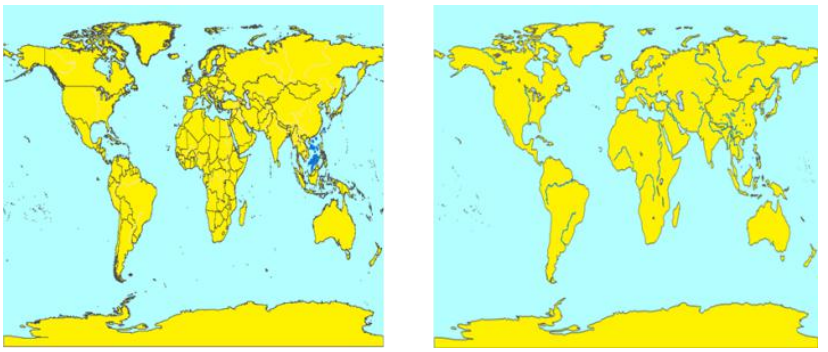

Figure 8.Overall Comparison Chart of Plate Generalization

Let us consider the integration of the small-scale measuring plates of the world map as an example of the proposed method. As shown in region A of Figure 9, in the original 1:5 million data, there are many small islands in the Canadian version of this map, which contains numerous small map spots and has a complex topography. After combination, at the 1:2.1 billion measuring scale, the small islands are combined and simplified to make the picture clearer, improving data coordination and 
summarizing greatly (as shown in Fig 9. A1 and A2 are in effect comparisons between the 1:5 million and 1:2.1 billion measuring scales, respectively, before and after integration). In region $\mathrm{B}$ of Figure 9 , to screen, summarize or simplify data according to specification and indicators of world map integration, to make the small-scale map data more reasonable, precise and complete (as shown in Fig 9. B1 and B2).

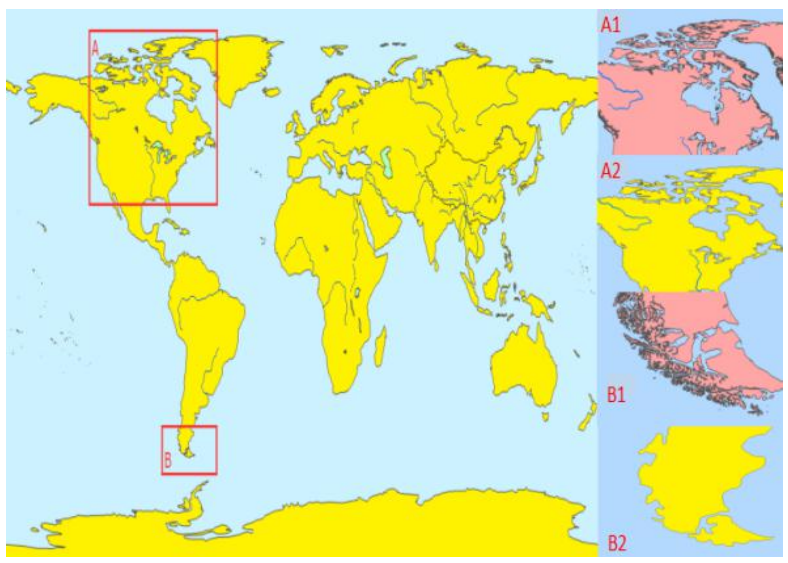

Figure 9.Local Comparison of Plate Generalization

\subsection{Comparison of Water-System Integrations}

The ultimate goal of water-system integration is to retain the large-area water systems and let them flow into the ocean, while merging small-area water systems with the original watersystem data to become the final tapered river line. In China, we keep the Yangtze River and the Yellow River. In other countries, we retain the second-order streams as well. We adopt a watersystem integration algorithm to ensure the same spatial relations and maintain the original stream shapes. The water system gradually turns to be more steady and reasonable, and is better than artificial gradient effect. In areas with dense river networks, the entire hierarchy is shown.

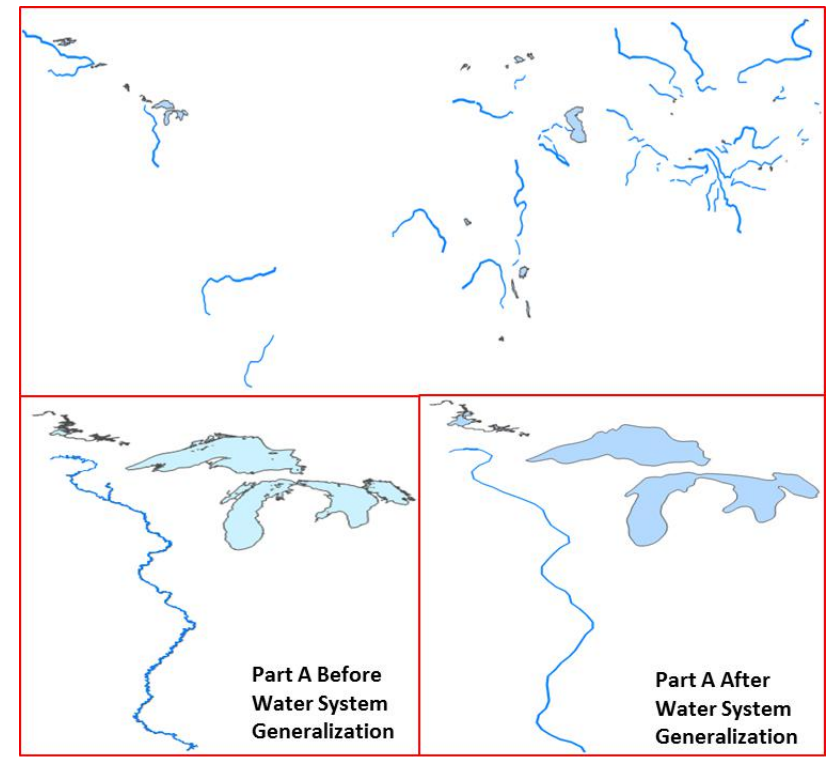

Figure. 10 Local Comparison of Water System Generalization

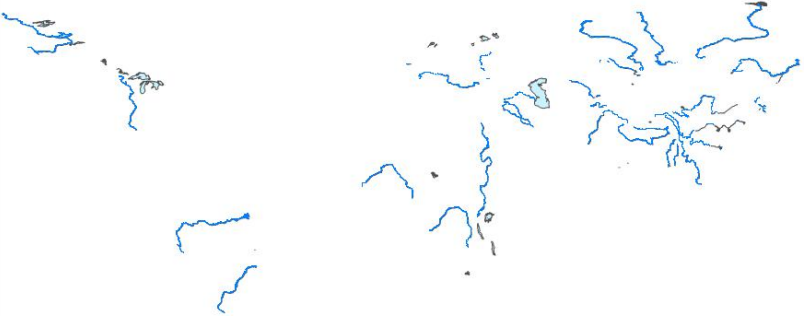

Figure. 11 Before Water System Generalization

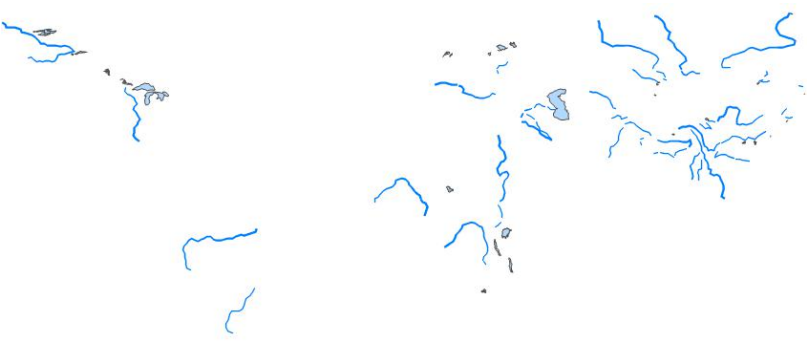

Figure.12 After Water System Generalization

\section{CONCLUSION}

Using the mapping work station, in this article, we have described the automated integration of original-scale worldwide map plates from a 1:5 million scale to a 1:200 million scale. In comparison with traditional drawing methods or half-automated integration methods, this model employs specific rules to : 1) Realize span measuring scale's generalization by steps or across steps. This is especially useful for integrating maps with a relatively large difference in measuring scales. 2) Provide high efficiency in comprehensive charting. Maps can be generated automatically, with cross-scale points, lines, and surface features. 3) Provide a flexible, comprehensive charting-tool set with rich annotation methods. One-touch operation can be obtained. 4) The auto-mapping knowledge engine is an intelligent platform system for fully automatic composite mapping. When handling politically sensitive islands, disconnecting or connecting islands, automatic machine recognition cannot be used, and manual handling is still needed. However, special islands can be marked with attributes or codes to enable subsequent automated processing.

Map integration is a very complicated process. Currently it is restricted by data source, demand, theoretical method, and scope of application, among others. Fully automatic integration technology still needs to be developed and perfected in mapping practice. The figure-number separation model used for the small-scale world-map integration described in this article can greatly improve the efficiency of integrating various scales, reduce manual labor in production, and solve aggregate problems in mapping, such as integration from traditional single measuring scales, to multiple scales, and across multiple scales. This approach has great significance for scale-free mappingintegration theory.

\section{ACKNOWLEDGEMENTS}

This work was supported by the National Key Research and Development Plan (Project Nos. 2016YFF0201305). 


\section{REFERENCES}

Ai,T., Guo,R.2000, A Constrained Delaunay Partitioning of Areal Objects to Support Map Generalization,Journay of Wuhan. Technical University of Surveying and Mapping,25(1),pp:35-41.

Chen,Y., Wang,H. 2015, Research and Implementation of Automatic Map Based on National Census Geography Information Data. Bulletin of surveying and mapping,(7),pp:106-108.

Feng,M., Lu,X., Cheng,M., Deng,An. 2017, A Method of Line Simplification Considering Patches Outline Considering Topological Relations, Bulletin of surveying and mapping,(6),pp:49-52.

Huang,Z., Qian,H., Guo,M., Liu,H., Wang,X. 2013, Matching Algorithm of Polygon Habitations Based on Their Skeletonlines Using Fourier Transform, Journal of surveying and mapping,42(6),pp:913-921.

Joachim,G., Herman,J., Marc,V. 2004, Constrained Higher Order Delaunay Triangulations, Elsevier Journal, 11(001),pp:271-277.

Li,L., Wang,J., Shen,D., Chen,Y. 2008, A Method For Plotting Gradual Change Symbol of Single Line Stream, Bulletin of surveying and mapping,(11),pp:64-67.

Liu,X., Wu,F., Hu,Y., Hu,H. 2013, A Method of Extracting Multiscale Skeletons for Polygonal Shapes, Journal of surveying and mapping,42(4),pp:588-594.

Liu, X., ZHAN,F., AI T. 2010, Road Selection Based on Voronoi Diagrams and 'Strokes' in Map Generalization. International Journal of Applied Earth Observation and Geoinformation, (12),pp:194-202.

Stoter,P., Van,A., Nijhuis.,Bruns. 2014, Fully Automated Generalization of a $1: 50 \mathrm{~K}$ map from 1:10k Data. Taylor journal,pp:1-13

Wu,W., Li,C., Yin,y., and Guo,P. 2016, An Automatic Plotting Algorithm of the Gradual Change of River Based on Directed Topology, Science of Surveying and Mapping,41(12),pp:89-93.

Yang,M., Ai,T., Zhou,Q., 2013, A Method of Road Network Generalization Considering Stroke Properties of Road Object, Journal of surveying and mapping,42(4),pp:49-52.

Yin,y. 2016, What is The Use of The WJ_III Map Workstation, China Academic Journal Electronic Publishing House,(3),pp:20-22.

Zhao,J. 2017, The Use of Database Mapping Technology in the Publication of Small-scale World Map, Bulletin of surveying and mapping,(3),pp:90-93. 